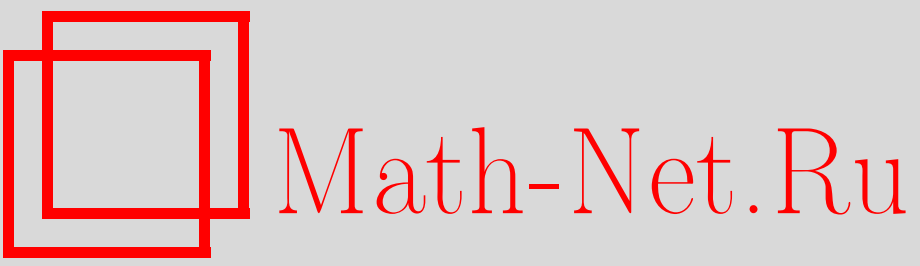

А. К. Аржников, А. А. Багрец, Д. А. Багрец, Усреднение резольвенты с коррелированным в пространстве распределением случайного потенциала, ТМФ, 1998, том 114, номер 2, 296-313

DOI: https://doi.org/10.4213/tmf841

Использование Общероссийского математического портала Math-Net.Ru подразумевает, что вы прочитали и согласны с пользовательским соглашением

http://www.mathnet.ru/rus/agreement

Параметры загрузки:

IP : 54.80 .73 .141

26 апреля 2023 г., 12:45:34 
ТЕОРЕТИЧЕСКАЯ

И МАТЕМАТИЧЕСКАЯ

ФИЗИКА

Том 114, № 2

февраль, 1998

(C) 1998 г. $\quad$ А.К. Аржников* , А. А. Багрец ${ }^{\dagger}$, Д. А. Багрец ${ }^{\dagger}$

\section{УСРЕДНЕНИЕ РЕЗОЛЬВЕНТЫ \\ С КОРРЕЛИРОВАННЫМ В ПРОСТРАНСТВЕ РАСПРЕДЕЛЕНИЕМ СЛУЧАЙНОГО ПОТЕНЦИАЛА}

Рассматривается задача о спектре квазичастиц бинарного неупорядоченного сплава с коррелированным в пространстве распределением случайного потенциала. Для представления усредненной резольвенты используется формализм расширенного пространства. Предложен ряд схем самосогласованных аппроксимаций для вычисления массового оператора, которые при $\alpha=0$ ( $\alpha$ - параметр ближнего порядка) совпадают с хорошо известными самосогласованными приближениями. При $\alpha \rightarrow 1$ для любых концентраций и $\alpha \rightarrow-1$ для концентрации $50 \%$ теория дает правильный предельный переход к функции Грина идеального кристалла. Аппроксимации обладают правильными аналитическими свойствами при любых значениях параметра $\alpha$.

\section{1. ВВЕДЕНИЕ}

Физика неупорядоченных систем часто сталкивается с проблемами вычисления усредненных физических величин при наличии пространственных корреляций. Примерами служат учет в спектре квазичастиц корреляций расположения атомов сплава и влияние корреляций спиновых и зарядовых плотностей на состояния магнетиков с коллективизированными электронами. Необходимо признать, что пока не удалось сформулировать единого принципа для описания неупорядоченных систем столь же мощного, как теорема Блоха для идеальных кристаллов.

Однако сушествует ряд методов, которые имеют достаточно высокий уровень математического обоснования, а приближения, получаемые в них, контролируются по малому параметру. K таким подходам прежде всего относится метод, базируюшийся на формализме расширенного пространства (ФРП) [1-4]. Первоначально ФРП возник для решения задач с независимыми распределениями случайных величин. В дальнейшем без серьезных изменений он использовался и для задач с коррелированным беспорядком [5-7]. Однако до конца удачными эти попытки назвать нельзя по ряду причин. В одних случаях нарушались аналитические свойства функции Грина, в других был невозможен контроль за сделанными приближениями либо область их применения была

\footnotetext{
* Физико-технический институт УрО РАН, Ижевск, Россия

$\dagger$ Удмуртский государственный университет, Ижевск, Россия
} 
ограничена малым по величине параметром ближнего порядка. В данной работе мы продолжаем развитие ФРП, но уже с учетом пространственных корреляций случайного потенциала.

Изучается модель бинарного сплава с диагональным беспорядком в приближении сильной связи. Гамильтониан имеет вид

$$
H\left(\left\{\epsilon_{\mathbf{n}}\right\}\right)=\sum_{\mathbf{i}}|\mathbf{i}\rangle \epsilon_{\mathbf{i}}\left\langle\mathbf{i}\left|+\sum_{\mathbf{i} \mathbf{j}}\right| \mathbf{i}\right\rangle V_{\mathbf{i}-\mathbf{j}}\langle\mathbf{j}|,
$$

здесь $|\mathbf{i}\rangle$ - вектор состояния электрона, локализованного на узле с радиус-вектором $\mathbf{i}$. Будем полагать, что множество векторов $\{|\mathbf{i}\rangle\}$ образует полный ортонормированный базис в пространстве $\Psi$. Конфигурация сплава описывается набором случайных величин $\left\{\epsilon_{\mathbf{i}}\right\}$, где $\epsilon_{\mathbf{i}}=\epsilon_{\mathbf{A}}$, если в узле $\mathbf{i}$ находится атом сорта $\mathbf{A}$, и $\epsilon_{\mathbf{i}}=\epsilon_{\mathbf{B}}$, если в узле $\mathbf{i}$ находится атом сорта $\mathbf{B}$. Для сплава с ближним порядком величины $\epsilon_{\mathbf{i}}$ не являются независимыми, т.е. $\epsilon_{\mathbf{i}}$ и $\epsilon_{\mathbf{j}}$ коррелируют на различных узлах решетки.

Усредненная функция Грина гамильтониана (1.1) определяется выражением

$$
\bar{G}_{\mathbf{i} \mathbf{j}}(\epsilon)=\iint \cdots \int\left\langle\mathbf{i}\left|\left[\epsilon-H\left(\left\{\epsilon_{\mathbf{n}}\right\}\right)\right]^{-1}\right| \mathbf{j}\right\rangle P\left(\left\{\epsilon_{\mathbf{n}}\right\}\right) \prod_{\mathbf{n}} d \epsilon_{\mathbf{n}},
$$

где $P\left(\left\{\epsilon_{\mathbf{n}}\right\}\right)$ - совместная функция распределения случайных величин, при этом

$$
P_{\epsilon_{\mathbf{i}}}(\epsilon)=x \delta\left(\epsilon-\epsilon_{\mathbf{A}}\right)+y \delta\left(\epsilon-\epsilon_{\mathbf{B}}\right)=\iint \cdots \int P\left(\left\{\epsilon_{\mathbf{n}}\right\}\right) \prod_{\mathbf{n} \neq \mathbf{i}} d \epsilon_{\mathbf{n}}
$$

есть плотность распределения случайной величины $\epsilon_{\mathbf{i}}$ на фиксированном узле. Здесь $x$ и $y$ - концентрации атомов сорта $\mathbf{A}$ и $\mathbf{B}$, соответственно. Интегралы перекрытия $V_{\mathbf{i}-\mathbf{j}}$ в гамильтониане (1.1) предполагаются неслучайными. Сначала рассматривается одномерный случай, результаты которого затем обобшаются в разделе 6 для произвольной размерности.

\section{2. КОРРЕЛЯЦИОННЫЕ ФУНКЦИИ}

Для построения $\Phi Р П$ с учетом пространственных корреляций необходимо знать корреляционные функции произведений случайных величин. Рассмотрим одномерную цепочку, конфигурация атомов $\mathbf{A}$ и $\mathbf{B}$ на которой определяется марковским процессом [8]. Введем следуюшие обозначения: пусть $P\left(s_{\mathbf{i}} \mid s_{\mathbf{j}}\right)-$ вероятность найти атом сорта $s_{\mathbf{i}}$ в узле $\mathbf{i}$ при условии, что в узле $\mathbf{j}$ находится атом сорта $s_{\mathbf{j}}, P\left(s_{\mathbf{i}}\right)$ - вероятность обнаружить атом $s_{\mathbf{i}}$ в узле $\mathbf{i}$. Вероятность образования кластера заданной конфигурации из $n$ атомов выражается через произведение парных условных вероятностей

$$
P_{n}\left(s_{\mathbf{l}_{1}}, s_{\mathbf{l}_{2}}, \ldots, s_{\mathbf{l}_{\mathbf{n}}}\right)=P\left(s_{\mathbf{l}_{1}}\right) P\left(s_{\mathbf{l}_{2}} \mid s_{\mathbf{l}_{1}}\right) P\left(s_{\mathbf{l}_{3}} \mid s_{\mathbf{l}_{2}}\right) \ldots P\left(s_{\mathbf{l}_{\mathbf{n}}} \mid s_{\mathbf{l}_{\mathrm{n}-1}}\right),
$$

где $l_{1}<l_{2}<\cdots<l_{n}$. Параметр Каули ближнего порядка есть

$$
\alpha=1-\frac{P^{\mathbf{A B}}}{x y},
$$


где $P^{\mathbf{A B}}$ - вероятность образования пары $\mathbf{A - B}$ на ближайших узлах. Будем считать, что $\mathbf{A}$ - примесный атом, т.е. $x<y$. В случае полного отталкивания между атомами разного сорта $P^{\mathrm{AB}}=0$. В противоположном случае максимального притяжения $P^{\mathrm{AB}}=x$. Таким образом, $0 \leq P^{\mathrm{AB}} \leq x,-x / y \leq \alpha \leq 1$. В отсутствие ближнего порядка $P^{\mathrm{AB}}=x y, \alpha=0$. Используя $(2.1),(2.2)$, нетрудно показать, что вероятность образования пары $\mathbf{A}-\mathbf{B}$ на расстоянии $n$ есть

$$
P_{n}^{\mathbf{A B}}=x y\left(1-\alpha^{n}\right)
$$

Следовательно, (2.1) выражается через $x$ и $\alpha$. Пусть

$$
\begin{aligned}
\bar{\epsilon} & =x \epsilon_{\mathbf{A}}+y \epsilon_{\mathbf{B}}, \\
\xi_{\mathbf{i}} & =\epsilon_{\mathbf{i}}-\bar{\epsilon}, \quad \bar{\xi}_{\mathbf{i}}=0 .
\end{aligned}
$$

Обозначим

$$
\Delta \xi=(y-x)\left(\epsilon_{\mathbf{A}}-\epsilon_{\mathbf{B}}\right), \quad \Delta=\sqrt{x y}\left(\epsilon_{\mathbf{A}}-\epsilon_{\mathbf{B}}\right) .
$$

Поскольку $\xi_{\mathbf{i}}$ принимает только два значения: $\xi_{\mathbf{A}}=\epsilon_{\mathbf{A}}-\bar{\epsilon}$ и $\xi_{\mathbf{B}}=\epsilon_{\mathbf{B}}-\bar{\epsilon}$, то $\left(\xi_{\mathbf{i}}-\xi_{\mathbf{A}}\right) \times$ $\left(\xi_{\mathbf{i}}-\xi_{\mathbf{B}}\right)=0$, откуда следует, что

$$
\xi_{\mathbf{i}}^{2}=(\Delta \xi) \xi_{\mathbf{i}}+\Delta^{2}
$$

Определим функции

$$
\begin{aligned}
\left\langle\xi_{\mathbf{l}_{1}}, \xi_{\mathbf{l}_{2}}, \ldots, \xi_{\mathbf{l}_{n}}\right\rangle_{c} & =\Delta^{2}(\Delta \xi)^{n-2} \alpha^{l_{n}-l_{1}}, \quad n \geq 2 \\
\left\langle\xi_{\mathbf{i}}\right\rangle_{c} & =0
\end{aligned}
$$

здесь и далее $l_{1}<l_{2}<\cdots<l_{n}$ и старшие степени $\xi_{\mathbf{i}}$ в силу (2.7) рассматривать не нужно. Тогда, используя (2.1), (2.3), получим

$$
\begin{aligned}
\left\langle\xi_{\mathbf{l}_{1}} \xi_{\mathbf{l}_{2}}\right\rangle & =\left\langle\xi_{\mathbf{l}_{1}} \xi_{\mathbf{l}_{2}}\right\rangle_{c}, \\
\left\langle\xi_{\mathbf{l}_{1}} \xi_{\mathbf{l}_{2}} \xi_{\mathbf{l}_{3}}\right\rangle & =\left\langle\xi_{\mathbf{l}_{1}} \xi_{\mathbf{l}_{2}} \xi_{\mathbf{l}_{3}}\right\rangle_{c}, \\
\left\langle\xi_{\mathbf{l}_{1}} \xi_{\mathbf{l}_{2}} \xi_{\mathbf{l}_{3}} \xi_{\mathbf{l}_{4}}\right\rangle & =\left\langle\xi_{\mathbf{l}_{1}} \xi_{\mathbf{l}_{2}}\right\rangle_{c}\left\langle\xi_{\mathbf{l}_{3}} \xi_{\mathbf{l}_{4}}\right\rangle_{c}+\left\langle\xi_{\mathbf{l}_{1}} \xi_{\mathbf{l}_{2}} \xi_{\mathbf{l}_{3}} \xi_{\mathbf{l}_{4}}\right\rangle_{c}, \\
\left\langle\xi_{\mathbf{l}_{1}} \xi_{\mathbf{l}_{2}} \xi_{\mathbf{l}_{3}} \xi_{\mathbf{l}_{4}} \xi_{\mathbf{l}_{5}}\right\rangle & =\left\langle\xi_{\mathbf{l}_{1}} \xi_{\mathbf{l}_{2}} \xi_{\mathbf{l}_{3}}\right\rangle_{c}\left\langle\xi_{\mathbf{l}_{4}} \xi_{\mathbf{l}_{5}}\right\rangle_{c}+\left\langle\xi_{\mathbf{l}_{1}} \xi_{\mathbf{l}_{2}}\right\rangle_{c}\left\langle\xi_{\mathbf{l}_{3}} \xi_{\mathbf{l}_{4}} \xi_{\mathbf{l}_{5}}\right\rangle_{c}+\left\langle\xi_{\mathbf{l}_{1}} \xi_{\mathbf{l}_{2}} \xi_{\mathbf{l}_{3}} \xi_{\mathbf{l}_{4}} \xi_{\mathbf{l}_{5}}\right\rangle_{c},
\end{aligned}
$$

где $\langle\ldots\rangle$ обозначает конфигурационное усреднение. Остальные многоузельные корреляционные функции определяются аналогичным образом.

\section{3. ПОСТРОЕНИЕ БАЗИСА В РАСШИРЕННОМ ПРОСТРАНСТВЕ}

Следуя $\Phi Р П[1,2,4]$, сопоставим вешественной случайной величине $\xi_{\mathbf{i}}(2.5)$ самосопряженный оператор $\hat{\xi}_{\mathbf{i}}$, действующий на вспомогательном пространстве $\Phi$. При этом множество значений случайной величины есть спектр изображаюшего ее оператора. В 
модели бинарного сплава $\xi_{\mathbf{i}}$ имеет распределение вида (1.3) и $\Phi$ является $2^{N}$-мерным линейным пространством, где $N$ - число узлов решетки. В пространстве $\Phi$ определен полный ортонормированный базис $\{|s\rangle\}$, где $s=\left(s_{1}, s_{2}, \ldots, s_{N}\right)$ и $s_{\mathbf{i}}$ есть или $\mathbf{A}$, или $\mathbf{B}$. Состояния $\{|s\rangle\}$ - это собственные векторы оператора $\hat{\xi}_{\mathbf{i}}$ для всех $\mathbf{i}$, при этом

$$
\hat{\xi}_{\mathbf{i}}|s\rangle=\xi_{\mathbf{i}}\left(s_{\mathbf{i}}\right)|s\rangle, \quad \xi_{\mathbf{i}}\left(s_{\mathbf{i}}\right)= \begin{cases}\epsilon_{\mathbf{A}}-\bar{\epsilon}, & s_{\mathbf{i}}=\mathbf{A} \\ \epsilon_{\mathbf{B}}-\bar{\epsilon}, & s_{\mathbf{i}}=\mathbf{B}\end{cases}
$$

Согласно этому определению $\hat{\xi}_{\mathbf{i}}$ коммутируют между собой.

Пусть теперь $f\left(\xi_{\mathbf{i}}, \xi_{\mathbf{j}}, \ldots, \xi_{\mathbf{k}}\right)$ - функция случайных величин $\xi_{\mathbf{i}}, \xi_{\mathbf{j}}, \ldots, \xi_{\mathbf{k}}$. Тогда соответствуюший ей оператор определяется как $\hat{f}=f\left(\hat{\xi}_{\mathbf{i}}, \hat{\xi}_{\mathbf{j}}, \ldots, \hat{\xi}_{\mathbf{k}}\right)$, а матричный элемент $\langle s|\hat{f}| s\rangle$ в силу (3.1) есть значение функции для фиксированной конфигурации атомов на цепочке. Пусть $p(s)$ - вероятность конфигурации $s$. Определим вектор основного состояния $|\mathrm{vac}\rangle \in \Phi$ следуюшим образом:

$$
|\mathrm{vac}\rangle=\sum_{s} \sqrt{p(s)}|s\rangle
$$

Тогда значение величины $f$, усредненное по всем конфигурациям системы, есть

$$
\bar{f}=\langle\operatorname{vac}|\hat{f}| \operatorname{vac}\rangle \text {. }
$$

Так же как в [3, 4], построим базис, состояший из векторов, которые порождают оператор $\hat{\xi}_{\mathbf{i}}$, действуя на состояние $|\mathrm{vac}\rangle$. В этом базисе пространству $\Phi$ внутренних степеней свободы можно будет придать структуру пространства Фока

$$
\Phi=E_{0} \oplus E_{1} \oplus \cdots \oplus E_{n} \oplus \cdots
$$

Здесь $E_{0}$ - одномерное пространство, порожденное вектором (3.2). Пространство $E_{1}$ размерности $N$, соответствуюшее состояниям с однократными возбуждениями, определим как линейную оболочку векторов

$$
|\mathbf{i}\rangle=\Delta^{-1} \hat{\xi}_{\mathbf{i}}|\mathrm{vac}\rangle,
$$

которые в отличие от случая независимых случайных величин не являются ортогональными друг другу. Из соотношений (2.8), (2.9) следует, что матрица Грама скалярного произведения векторов (3.5) есть $g_{i j}=\{\mathbf{i} \mid \mathbf{j}\}=\alpha^{|i-j|}$. Ее фурье-образ равен

$$
g(q)=\frac{1}{N} \sum_{i j} g_{i j} \exp \{i q(i-j)\}=\frac{1-\alpha^{2}}{1+\alpha^{2}-2 \alpha \cos q}, \quad q \in[-\pi, \pi]
$$

Если $|\alpha|<1$, то $g(q)>0$, следовательно, матрица $g_{i j}$ положительно определена, поэтому совокупность векторов (3.5) линейно независима и образует полный базис в $E_{1}$. Поскольку $\bar{\xi}_{\mathbf{i}}=\left\langle\operatorname{vac}\left|\hat{\xi}_{\mathbf{i}}\right| \operatorname{vac}\right\rangle=0$, то $E_{1}$ ортогонально $E_{0}$. 
Определим векторы $|\mathbf{i}, \mathbf{j}\rangle(i<j)$ пространства $E_{2}$ таким образом, что

$$
\langle\operatorname{vac} \mid \mathbf{i}, \mathbf{j}\rangle=0, \quad\left\langle\mathbf{i}^{\prime} \mid \mathbf{i}, \mathbf{j}\right\rangle=0
$$

для любых $\mathbf{i}^{\prime}$ и $\mathbf{i} \neq \mathbf{j}$. Пусть

$$
b_{1}|\mathbf{i}, \mathbf{i}+\mathbf{1}\rangle=\left(\hat{\xi}_{\mathbf{i}+1} \hat{\xi}_{\mathbf{i}}-a_{0}-a_{1}\left(\hat{\xi}_{\mathbf{i}}+\hat{\xi}_{\mathbf{i}+1}\right)\right)|\operatorname{vac}\rangle .
$$

Поскольку вектор $|\mathbf{i}, \mathbf{i}+\mathbf{1}\rangle$ должен удовлетворять соотношениям (3.7), а вектор $|\mathbf{i}\rangle$ определяется $(3.5)$, то, действуя $\langle\operatorname{vac}|$ и $\langle\operatorname{vac}| \hat{\xi}_{\mathbf{i}}$ слева на $(3.8)$ и учитьвая $(2.8),(2.9)$, находим коэффициенты

$$
a_{0}=\Delta^{2} \alpha, \quad a_{1}=\Delta \xi \frac{\alpha}{1+\alpha} .
$$

Коэффициент $b_{1}$ в (3.8) определяется из условия нормировки

$$
b_{1}^{2}=\Delta^{4}\left(1-\alpha^{2}\right)+\Delta^{2}(\Delta \xi)^{2} \frac{1-\alpha}{1+\alpha} \alpha .
$$

Так как $b_{1}^{2}>0$, то из (3.9) следует отмеченное ранее ограничение на параметр Каули $-x / y<\alpha<1(x<y)$. При $\alpha=1$ либо при $\alpha=-x / y$ получаем, что $b_{1}=0$. Поскольку корреляционные функции $(2.8),(2.9)$ зависят от параметра $\alpha$ степенным образом, можно убедиться в том, что построенньй согласно (3.8) вектор $|\mathbf{i}, \mathbf{i}+\mathbf{1}\rangle$ ортогонален не только $|\mathbf{i}\rangle$ и $|\mathbf{i}+\mathbf{1}\rangle$, но и всем векторам $|\mathbf{j}\rangle \in E_{1}$. Кроме того, $\langle\mathbf{i}, \mathbf{i}+\mathbf{1} \mid \mathbf{j}, \mathbf{j}+\mathbf{1}\rangle=0$, если $\mathbf{i} \neq \mathbf{j}$.

Для произвольного вектора $|\mathbf{i}, \mathbf{i}+\mathbf{n}\rangle$ имеем

$$
b_{n}|\mathbf{i}, \mathbf{i}+\mathbf{n}\rangle=\left(\hat{\xi}_{\mathbf{i}} \hat{\xi}_{\mathbf{i}+\mathbf{n}}-L_{n+1}^{(1)}\left(\hat{\xi}_{\mathbf{i}}, \hat{\xi}_{\mathbf{i}+\mathbf{1}}, \ldots, \hat{\xi}_{\mathbf{i}+\mathbf{n}}\right)\right)|\operatorname{vac}\rangle
$$

Здесь $L_{n}^{(1)}$ - полином от $n$ переменных степени 1. Его коэффициенты находятся, как и ранее, из условия ортогональности и нормировки. Определим пространство $E_{2}$ как линейную оболочку векторов $\{|\mathbf{i}, \mathbf{i}+\mathbf{n}\rangle\}$ вида (3.10). При условии $-x / y<\alpha<1$ можно показать, что совокупность векторов (3.10) образует полный (но не ортогональный) базис в $E_{2}$. При этом для пары векторов $|\mathbf{i}, \mathbf{j}\rangle(j>i)$ и $|\mathbf{l}, \mathbf{m}\rangle(m>l)$ имеем

$$
\begin{array}{ll}
\langle\mathbf{i}, \mathbf{j} \mid \mathbf{l}, \mathbf{m}\rangle=0, & j \leq l, \\
\langle\mathbf{i}, \mathbf{j} \mid \mathbf{l}, \mathbf{m}\rangle=\frac{b_{j-l}^{2}}{b_{j-i} b_{m-l}} \alpha^{l-i} \alpha^{m-j}, & i \leq l<j \leq m, \\
\langle\mathbf{i}, \mathbf{j} \mid \mathbf{l}, \mathbf{m}\rangle=\frac{b_{m-l}}{b_{j-i}} \alpha^{l-i} \alpha^{j-m}, & i \leq l<m \leq j .
\end{array}
$$

Построенное таким образом пространство $E_{2}$ ортогонально $E_{0}$ и $E_{1}$.

В обшем случае пространству $E_{n}$ соответствуют векторы

$$
\left|\sigma_{n}(\mathbf{l})\right\rangle=\left|\mathbf{l}_{1}, \mathbf{l}_{2}, \ldots, \mathbf{l}_{n}\right\rangle,
$$


где $\sigma_{n}$ - конфигурация кластера, $\mathbf{l}$ - положение его центра тяжести, $l_{1}<l_{2}<\cdots<l_{n}$. По аналогии с соотношением (3.10) запишем

$$
\begin{aligned}
b_{\sigma_{n}}\left|\mathbf{l}_{1}, \mathbf{l}_{2}, \ldots, \mathbf{l}_{n}\right\rangle= & \left(\hat{\xi}_{\mathbf{l}_{1}} \hat{\xi}_{\mathbf{l}_{2}} \ldots \hat{\xi}_{\mathbf{l}_{n}}-\right. \\
& \left.-L_{l_{n}-l_{1}+1}^{(n-1)}\left(\hat{\xi}_{\mathbf{l}_{1}}, \hat{\xi}_{\mathbf{l}_{1}+\mathbf{1}}, \hat{\xi}_{\mathbf{l}_{1}+\mathbf{2}}, \ldots, \xi_{\mathbf{l}_{n}}\right)\right)|\operatorname{vac}\rangle .
\end{aligned}
$$

Здесь $L_{k}^{(n-1)}\left(\xi_{1}, \xi_{2}, \ldots, \xi_{k}\right), n \leq k,-$ полином от $k$ переменных обшей степени $(n-1)$ вида

$$
\begin{aligned}
L_{k}^{(n-1)}\left(\xi_{1}, \xi_{2}, \ldots, \xi_{k}\right)= & c_{0}^{(n)}+\sum_{i=1}^{k} c_{i}^{(n)} \xi_{i}+\sum_{1 \leq i<j \leq k} c_{i j}^{(n)} \xi_{i} \xi_{j}+\cdots \\
& \cdots+\sum_{1 \leq i_{1}<i_{2}<\cdots<i_{n-1} \leq k} c_{i_{1} i_{2} \ldots i_{n-1}}^{(n)} \xi_{i_{1}} \xi_{i_{2}} \ldots \xi_{i_{n-1}}
\end{aligned}
$$

Коэффициенты полинома (3.14) определяются из условия ортогональности вектора $\left|\sigma_{n}(\mathbf{l})\right\rangle$ любому кластеру $\sigma_{m}\left(\mathbf{l}^{\prime}\right) \subseteq\left\{\mathbf{l}_{1}, \mathbf{l}_{2}, \ldots, \mathbf{l}_{n}\right\}(m<n)$ с помошью соотношений $(2.7)$ $(2.8)$ и (2.9). Тогда построенный вектор $\left|\sigma_{n}(\mathbf{l})\right\rangle$ будет ортогонален всем векторам $|s\rangle \in$ $E_{0} \oplus E_{1} \oplus \cdots \oplus E_{n-1}$. Различные векторы $\left|\sigma_{n}(\mathbf{l})\right\rangle$ и $\left|\sigma_{n}^{\prime}\left(\mathbf{l}^{\prime}\right)\right\rangle$ ортогональны, если кластеры $\sigma_{n}(\mathbf{l})$ и $\sigma_{n}^{\prime}\left(\mathbf{l}^{\prime}\right)$ не перекрываются. Иначе скалярные произведения отличны от нуля подобно (3.11).

В представлении ФРП гамильтониан (1.1) имеет вид [4]

$$
\mathcal{H}=\sum_{\mathbf{i}}|\mathbf{i}\rangle\left\langle\mathbf{i}\left|\otimes \hat{\xi}_{\mathbf{i}}+\sum_{\mathbf{i} \mathbf{j}}\right| \mathbf{i}\right\rangle\left(\bar{\epsilon} \delta_{\mathbf{i} \mathbf{j}}+V_{\mathbf{i}-\mathbf{j}}\right)\langle\mathbf{j}| \otimes \hat{I}
$$

где $\hat{I}$ - единичный оператор на пространстве $\Phi$. Гамильтониан (3.15) действует в расширенном пространстве $\Psi \otimes \Phi,|\mathbf{i}\rangle \in \Psi$, которому, как и ранее, можно придать структуру пространства Фока:

$$
\Psi \otimes \Phi=E^{(0)} \oplus E^{(1)} \oplus \cdots \oplus E^{(n)} \oplus \cdots,
$$

где $E^{(n)}=\Psi \otimes E_{n}$. Базисный вектор в $E^{(n)}$ обозначим

$$
\left|\mathbf{i}+\mathbf{l}, \sigma_{n}(\mathbf{l})\right\rangle=|\mathbf{i}+\mathbf{l}\rangle \otimes\left|\sigma_{n}(\mathbf{l})\right\rangle
$$

здесь $\mathbf{i}$ - положение электрона, отсчитанное от центра тяжести кластера $\sigma_{n}(\mathbf{l})$, причем $\mathbf{i} \equiv 0$ при $n=0$. Согласно (3.13) имеем

$$
\operatorname{Im} \hat{\xi}_{\mathbf{i}}\left(E_{n}\right) \subseteq E_{n-1} \oplus E_{n} \oplus E_{n+1},
$$

поэтому в базисе (3.16) гамильтониан (3.15) имеет блочно-трехдиагональный вид

$$
\mathcal{H}_{n m}=\mathcal{H}_{n} \delta_{n m}+\Delta_{n} \delta_{n+1, m}+\Delta_{n}^{+} \delta_{n-1, m}
$$

где $\mathcal{H}_{n m}=P_{n} \mathcal{H} P_{m}, \Delta_{n}=P_{n} \mathcal{H} P_{n+1}$ и $P_{n}(n \geq 0)$ - ортогональный проектор на пространство $E^{(n)}$. 


\section{4. КОНФИГУРАЦИОННОЕ УСРЕДНЕНИЕ РЕЗОЛЬВЕНТЫ, САМОСОГЛАСОВАННЫЙ ПОДХОД}

В формализме расширенного пространства задача о нахождении функции Грина (1.2) сводится к вычислению проекции

$$
G_{0}(\epsilon)=P_{0} \frac{1}{\epsilon-\mathcal{H}} P_{0}
$$

резольвенты гамильтониана (3.15) на пространство $E^{(0)}$. Согласно (3.3) имеем

$$
G_{0}(\epsilon)=\sum_{\mathbf{l m}}\left|\mathbf{l}, \sigma_{0}(\mathbf{l})\right\rangle \bar{G}_{\mathbf{l m}}(\epsilon)\left\langle\mathbf{m}, \sigma_{0}(\mathbf{m})\right| .
$$

Для дальнейших вычислений удобно определить в пространстве $\Phi$ базис $\left\{\left|\chi\left(\sigma_{n}(\mathbf{l})\right)\right\rangle\right\}$, дуальный базису $\left\{\left|\sigma_{n}(\mathbf{1})\right\rangle\right\}$ (см. (3.12), (3.13)), такой, что

$$
\left\langle\chi\left(\sigma_{n}(\mathbf{l})\right) \mid \sigma_{m}^{\prime}\left(\mathbf{l}^{\prime}\right)\right\rangle=\delta_{\sigma \sigma^{\prime}} \delta_{n m} \delta_{1 \mathbf{l}^{\prime}} .
$$

В расширенном пространстве $\Psi \otimes \Phi$ возникает базис

$$
\left|\mathbf{i}+\mathbf{l}, \chi\left(\sigma_{n}(\mathbf{l})\right)\right\rangle=|\mathbf{i}+\mathbf{l}\rangle \otimes\left|\chi\left(\sigma_{n}(\mathbf{l})\right)\right\rangle,
$$

дуальный базису (3.16), т.е.

$$
\left\langle\mathbf{i}+\mathbf{l}, \chi\left(\sigma_{n}(\mathbf{l})\right) \mid \mathbf{j}+\mathbf{l}^{\prime}, \sigma_{m}^{\prime}\left(\mathbf{l}^{\prime}\right)\right\rangle=\delta_{\sigma \sigma^{\prime}} \delta_{n m} \delta_{\mathbf{l}^{\prime}} \delta_{\mathbf{i j}} .
$$

Перейдем к фурье-представлению:

$$
\begin{gathered}
\left|\mathbf{i}, \sigma_{n}, \mathbf{q}\right\rangle=\frac{1}{\sqrt{N}} \sum_{\mathbf{l}}\left|\mathbf{i}+\mathbf{l}, \sigma_{n}(\mathbf{l})\right\rangle \exp \{-i \mathbf{q} \mathbf{l}\}, \quad \mathbf{q} \in[-\pi, \pi], \\
\left|\chi\left(\mathbf{i}, \sigma_{n}, \mathbf{q}\right)\right\rangle=\frac{1}{\sqrt{N}} \sum_{\mathbf{l}}\left|\mathbf{i}+\mathbf{l}, \chi\left(\sigma_{n}(\mathbf{l})\right)\right\rangle \exp \{-i \mathbf{q} \mathbf{l}\}, \\
\left\langle\chi\left(\mathbf{i}, \sigma_{n}, \mathbf{q}\right) \mid \mathbf{j}, \sigma_{m}^{\prime}, \mathbf{q}^{\prime}\right\rangle=\delta_{\sigma \sigma^{\prime}} \delta_{n m} \delta_{\mathbf{i j}} \delta_{\mathbf{q q}}, \\
\left\langle\mathbf{i}, \sigma_{n}, \mathbf{q} \mid \mathbf{j}, \sigma_{m}^{\prime}, \mathbf{q}^{\prime}\right\rangle=\delta_{\mathbf{q} \mathbf{q}^{\prime}} \delta_{n m} \exp \{i \mathbf{q}(\mathbf{j}-\mathbf{i})\}\left\langle\sigma_{n}(\mathbf{i}) \mid \sigma_{n}^{\prime}(\mathbf{j})\right\rangle .
\end{gathered}
$$

Тогда гамильтониан (3.15) принимает вид

$$
\begin{aligned}
& \mathcal{H}=\sum_{\mathbf{q}} \mathcal{H}(\mathbf{q}), \quad \mathcal{H}(\mathbf{q})=\mathcal{H}_{\mathrm{vc}}(\mathbf{q})+\mathcal{U}(\mathbf{q}), \\
& \mathcal{H}_{\mathrm{vc}}(\mathbf{q})=\left|\mathbf{0}, \sigma_{0}, \mathbf{q}\right\rangle(\bar{\epsilon}+V(\mathbf{q}))\left\langle\mathbf{0}, \sigma_{0}, \mathbf{q}\right|+ \\
& +\sum_{\mathbf{i}, \mathbf{j}, \sigma_{n}, n \geq 1}\left|\mathbf{i}, \sigma_{n}, \mathbf{q}\right\rangle\left(\bar{\epsilon} \delta_{\mathbf{i j}}+V_{\mathbf{i}-\mathbf{j}}\right)\left\langle\chi\left(\mathbf{j}, \sigma_{n}, \mathbf{q}\right)\right|, \\
& V(\mathbf{q})=\frac{1}{N} \sum_{\mathbf{i} \mathbf{j}} V_{\mathbf{i}-\mathbf{j}} \exp \{i \mathbf{q}(\mathbf{i}-\mathbf{j})\}, \\
& \mathcal{U}(\mathbf{q})=\sum_{\mathbf{i}, \mathbf{d}, \sigma_{n}, \sigma_{m}^{\prime}}\left|\mathbf{i}, \sigma_{n}, \mathbf{q}\right\rangle \exp \{i \mathbf{q d}\} \xi_{\sigma_{n}(\mathbf{i}) \sigma_{m}^{\prime}(\mathbf{i}+\mathbf{d})}^{(\mathbf{0})}\left\langle\chi\left(\mathbf{i}+\mathbf{d}, \sigma_{m}^{\prime}, \mathbf{q}\right)\right|,
\end{aligned}
$$


где $\xi_{\sigma_{n}(\mathbf{i}) \sigma_{m}^{\prime}(\mathbf{i}+\mathbf{d})}^{(\mathbf{0})}=\left\langle\chi\left(\sigma_{n}(\mathbf{i})\right)\left|\hat{\xi}_{\mathbf{0}}\right| \sigma_{m}^{\prime}(\mathbf{i}+\mathbf{d})\right\rangle, \mathbf{d}-$ вектор, соединяющий центры тяжести кластеров $\sigma_{n}(\mathbf{i})$ и $\sigma_{m}^{\prime}(\mathbf{i}+\mathbf{d}), \mathcal{H}_{\mathrm{vc}}(\mathbf{q})$ - гамильтониан виртуального кристалла, $\mathcal{U}(\mathbf{q})-$ представление случайного потенциала.

Введем ортогональные проекторы на пространства $E_{\mathbf{q}}^{(n)}$ :

$$
P_{n}^{\mathbf{q}}=\sum_{\mathbf{i}, \sigma_{n}}\left|\mathbf{i}, \sigma_{n}, \mathbf{q}\right\rangle\left\langle\chi\left(\mathbf{i}, \sigma_{n}, \mathbf{q}\right)\right| .
$$

Используя их, (4.1) можно переписать в виде [4]

$$
G_{0}(\epsilon, \mathbf{q})=\frac{1}{\epsilon-\mathcal{H}_{0}(\mathbf{q})-\Sigma_{0}(\epsilon, \mathbf{q})}
$$

где

$$
\begin{aligned}
\mathcal{H}_{0}(\mathbf{q}) & =P_{0}^{\mathbf{q}} \mathcal{H}(\mathbf{q}) P_{0}^{\mathbf{q}}, \quad Q_{0}^{\mathbf{q}}=1-P_{0}^{\mathbf{q}}, \\
\Sigma_{0}(\epsilon, \mathbf{q}) & =P_{0}^{\mathbf{q}} \mathcal{H}(\mathbf{q}) Q_{0}^{\mathbf{q}} \frac{1}{\epsilon-Q_{0}^{\mathbf{q}} \mathcal{H}(\mathbf{q}) Q_{0}^{\mathbf{q}}} Q_{0}^{\mathbf{q}} \mathcal{H}(\mathbf{q}) P_{0}^{\mathbf{q}} .
\end{aligned}
$$

Продолжая проецирование, запишем $\Sigma_{0}(\epsilon, \mathbf{q})$ в виде цепной дроби [4]:

$$
\Sigma_{0}(\epsilon, \mathbf{q})=\Delta_{0} \frac{1}{\epsilon-\mathcal{H}_{1}-\Delta_{1} \frac{1}{\epsilon-\mathcal{H}_{2}-\cdot{ }_{-\Delta_{n-1} \frac{1}{\epsilon-\mathcal{H}_{n}-\Sigma_{n}} \Delta_{n-1}^{+}}} \Delta_{1}^{+}} \Delta_{0}^{+},
$$

где $\mathcal{H}_{n}(\mathbf{q})=P_{n}^{\mathbf{q}} \mathcal{H}(\mathbf{q}) P_{n}^{\mathbf{q}}, \Delta_{n}(\mathbf{q})=P_{n}^{\mathbf{q}} \mathcal{U}(\mathbf{q}) P_{n+1}^{\mathbf{q}}$.

Аналитическое вычисление формально точного выражения (4.6) невозможно из-за входяших в него матриц бесконечных размерностей. В отличие от [4] проблемы вычисления возникают уже на первом этаже цепной дроби из-за неортогональности векторов. Поэтому в каждом из пространств $E_{\mathbf{q}}^{(n)}$ удобно выбрать специальным образом ортонормированный базис. Далее вместо $\left|\mathbf{i}, \sigma_{n}, \mathbf{q}\right\rangle$ будем писать $\left|\sigma_{n}(\mathbf{i}), \mathbf{q}\right\rangle=\left|\mathbf{l}_{1}, \mathbf{l}_{2}, \ldots, \mathbf{l}_{n}, \mathbf{q}\right\rangle$, где $\sigma_{n}(\mathbf{i})=\left\{\mathbf{l}_{1}, \mathbf{l}_{2}, \ldots, \mathbf{l}_{n}\right\}$, как и прежде в (3.12), обозначает кластер конфигурации $\sigma_{n}$ с центром тяжести i. Ортогональный базис $\left\{\left|\psi_{m}, \mathbf{q}\right\rangle\right\}$ в $E_{\mathbf{q}}^{(1)}$ определим следуюшим образом:

$$
\begin{aligned}
&\left|\psi_{0}, \mathbf{q}\right\rangle=|\mathbf{0}, \mathbf{q}\rangle, \\
&\left|\psi_{m}, \mathbf{q}\right\rangle=\left(1-\alpha^{2}\right)^{-1 / 2}(|\mathbf{m}, \mathbf{q}\rangle-|\mathbf{m}-\mathbf{1}, \mathbf{q}\rangle \alpha \exp \{i q\}), \\
&\left|\psi_{-m}, \mathbf{q}\right\rangle=\left(1-\alpha^{2}\right)^{-1 / 2}(|-\mathbf{m}, \mathbf{q}\rangle-|-\mathbf{m}+\mathbf{1}, \mathbf{q}\rangle \alpha \exp \{-i q\}), \quad m>0 \\
&\left\langle\psi_{m}, \mathbf{q} \mid \psi_{k}, \mathbf{q}\right\rangle=\delta_{m k} .
\end{aligned}
$$

В пространстве $E_{\mathbf{q}}^{(2)}$ базисные векторы $\left|\psi_{m, m+1}, \mathbf{q}\right\rangle=|\mathbf{m}, \mathbf{m}+\mathbf{1}, \mathbf{q}\rangle$ ортогональны друг другу. Используя правило вычисления скалярных произведений (3.11), произвольный базисный вектор $\left|\psi_{m, m+k}, \mathbf{q}\right\rangle$ можно получить путем ортогонализации вектора $|\mathbf{m}, \mathbf{m}+\mathbf{k}, \mathbf{q}\rangle$ всем двукратным возбуждениям, входящим в сегмент $[m, m+k]$. 
Пусть $\mathcal{U}_{n}(\mathbf{q})=P_{n}^{\mathbf{q}} \mathcal{U}(\mathbf{q}) P_{n}^{\mathbf{q}}$. Согласно (3.17) гамильтониан (4.3) имеет блочно-трехдиагональный вид, поэтому

$$
\mathcal{U}(\mathbf{q})=\sum_{n \geq 1} \mathcal{U}_{n}(\mathbf{q})+\sum_{n \geq 0}\left(\Delta_{n}(\mathbf{q})+\Delta_{n}^{+}(\mathbf{q})\right)
$$

В ортогональном базисе матричные элементы случайного потенциала (4.8) можно вычислить, используя соотношение

$$
\left\langle\mathbf{i}, \sigma_{n}, \mathbf{q}|\mathcal{U}(\mathbf{q})| \mathbf{j}, \sigma_{m}^{\prime}, \mathbf{q}\right\rangle=\left\langle\sigma_{n}(\mathbf{i})\left|\hat{\xi}_{\mathbf{0}}\right| \sigma_{m}^{\prime}(\mathbf{j})\right\rangle \exp \{i \mathbf{q}(\mathbf{j}-\mathbf{i})\}
$$

Тогда имеем

$$
\left\langle\mathbf{0}, \sigma_{0}, \mathbf{q}\left|\Delta_{0}(\mathbf{q})\right| \psi_{m}, \mathbf{q}\right\rangle=\delta_{m, 0} \Delta .
$$

В пространстве $E_{\mathbf{q}}^{(1)}$ случайный потенциал $\mathcal{U}_{1}(\mathbf{q})$ диагонален в базисе $(4.7)$, т.е.

$$
\begin{aligned}
& \left\langle\psi_{0}, \mathbf{q}\left|\mathcal{U}_{1}(\mathbf{q})\right| \psi_{0}, \mathbf{q}\right\rangle=\Delta \xi \\
& \left\langle\psi_{m}, \mathbf{q}\left|\mathcal{U}_{1}(\mathbf{q})\right| \psi_{k}, \mathbf{q}\right\rangle=\delta_{m k} \Delta \xi \frac{\alpha^{|m|}}{1+\alpha}, \quad m \neq 0 .
\end{aligned}
$$

В отсутствие ближнего порядка $(\alpha=0)$ отличные от нуля матричные элементы $\Delta_{1}(\mathbf{q})$ равны [4]

$$
\left\langle\psi_{m}, \mathbf{q}\left|\Delta_{1}(\mathbf{q})\right| \psi_{0, m}, \mathbf{q}\right\rangle=\Delta \exp \{-i q m / 2\}, \quad m \neq 0 .
$$

При $\alpha \neq 0$ константа $\Delta$ в (4.11) заменяется другой константой, например

$$
\begin{aligned}
\left\langle\psi_{1}, \mathbf{q}\left|\Delta_{1}(\mathbf{q})\right| \psi_{0,1}, \mathbf{q}\right\rangle & =\Delta^{-1}\left(1-\alpha^{2}\right)^{-1 / 2} b_{1} \exp \{-i q / 2\}, \\
\left\langle\psi_{-1}, \mathbf{q}\left|\Delta_{1}(\mathbf{q})\right| \psi_{0,-1}, \mathbf{q}\right\rangle & =\Delta^{-1}\left(1-\alpha^{2}\right)^{-1 / 2} b_{1} \exp \{i q / 2\}
\end{aligned}
$$

где $b_{1}>0$ определяется условием (3.9). Так как векторы $\left|\psi_{m, m+k}\right\rangle$ построены путем ортогонализации векторов вида (3.10), то при $\alpha \neq 0$ отличны от нуля также и элементы $\left\langle\psi_{k}\left|\Delta_{1}(\mathbf{q})\right| \psi_{m, k}\right\rangle,\left\langle\psi_{-k}\left|\Delta_{1}(\mathbf{q})\right| \psi_{-m,-k}\right\rangle$, где $0<m<k$. Эти элементы обрашаются в нуль при $\alpha \rightarrow 0$, все остальные матричные элементы $\Delta_{1}(\mathbf{q})$ равны нулю.

В пространстве $E_{\mathbf{q}}^{(2)}$ случайный потеншиал $\mathcal{U}_{2}(\mathbf{q})$ диагонален по кластерам длины 1:

$$
\begin{aligned}
\left\langle\psi_{m, m+1}\left|\mathcal{U}_{2}(\mathbf{q})\right| \psi_{k, k+1}\right\rangle & =\delta_{m k} \frac{\Delta \xi}{1+\alpha} \alpha^{m}, \\
\left\langle\psi_{-m,-m-1}\left|\mathcal{U}_{2}(\mathbf{q})\right| \psi_{-k,-k-1}\right\rangle & =\delta_{m k} \frac{\Delta \xi}{1+\alpha} \alpha^{|m|}, \quad m \geq 0 .
\end{aligned}
$$

В общем случае матричные элементы $\left\langle\psi_{n, n+m}\left|\mathcal{U}_{2}(\mathbf{q})\right| \psi_{k, k+l}\right\rangle \neq 0$ и убывают степенным образом по параметру $\alpha$ подобно (4.13), если увеличивается расстояние между узлом 0 и центром тяжести кластера, соответствуюшего вектору $\left|\psi_{n, n+m}\right\rangle$ либо вектору $\left|\psi_{k, k+l}\right\rangle$.

Для дальнейшего упрощения необходимо использовать аппроксимационную схему, возможные варианты которой обсуждаются ниже. 
Выделим кластер $\sigma_{n}$, содержащий $n$ узлов, рассеяние на котором будем учитывать точно. В дальнейшем назовем его максимальным кластером $\sigma_{n}^{\max }$. Определим в пространстве $E_{\mathbf{q}}^{(1)} \oplus E_{\mathbf{q}}^{(2)} \oplus \cdots \oplus E_{\mathbf{q}}^{(n)}$ подпространство $L_{\mathbf{q}}=L_{\mathbf{q}}^{(1)} \oplus L_{\mathbf{q}}^{(2)} \oplus \cdots \oplus L_{\mathbf{q}}^{(n)}$, где $L_{\mathbf{q}}^{(m)}$ - линейная оболочка векторов $\left|\mathbf{i}, \sigma_{m}, \mathbf{q}\right\rangle$ таких, что $\mathbf{i} \cup \sigma_{m} \subseteq \sigma_{n}^{\max }(m \leq n)$. Из (3.13) нетрудно видеть, что $\mathcal{U}(\mathbf{q})$ отображает $L_{\mathbf{q}}$ на $L_{\mathbf{q}} \oplus E_{\mathbf{q}}^{(0)}$. Пусть $T_{m}^{\mathbf{q}}$ - ортогональный проектор на подпространство $L_{\mathbf{q}}^{(m)}$ и

$$
T^{\mathbf{q}}=\sum_{m=1}^{n} T_{m}^{\mathbf{q}}
$$

- ортогональный проектор на $L_{\mathbf{q}}$. В этих обозначениях несамосогласованная аппроксимация состоит в следующей замене:

$$
\mathcal{U}(\mathbf{q}) \Rightarrow T^{\mathbf{q}} \mathcal{U}(\mathbf{q}) T^{\mathbf{q}+}+\Delta_{0}(\mathbf{q})+\Delta_{0}^{+}(\mathbf{q})
$$

т.е. действие $\mathcal{U}(\mathbf{q})$ сужается на пространство $L_{\mathbf{q}} \oplus E_{\mathbf{q}}^{(0)}$. После замены (4.14) матрищы операторов $\Delta_{m}(\mathbf{q})$ и $\mathcal{U}_{m}(\mathbf{q})(m \leq n)$ имеют ненулевые элементы для конечного числа кластеров, поэтому обрашение матриц в (4.6) может быть выполнено аналитически.

Для построения самосогласованных аппроксимаций определим, следуя работе [4], операторы

$$
\begin{aligned}
& \Sigma^{\mathrm{eff}}(\epsilon, \mathbf{q})=\sum_{\mathbf{i}, \mathbf{j}, \sigma_{n}, n \geq 1}\left|\mathbf{i}, \sigma_{n}, \mathbf{q}\right\rangle \Sigma_{\mathbf{i}-\mathbf{j}}(\epsilon)\left\langle\chi\left(\mathbf{j}, \sigma_{n}, \mathbf{q}\right)\right|, \\
& \mathcal{H}^{\mathrm{eff}}(\epsilon, \mathbf{q})=\mathcal{H}_{\mathrm{vc}}(\mathbf{q})+\Sigma^{\mathrm{eff}}(\epsilon, \mathbf{q}),
\end{aligned}
$$

где $\Sigma_{\mathbf{i}-\mathbf{j}}(\epsilon)$ есть фурье-прообраз $\Sigma_{0}(\epsilon, \mathbf{q})$. Заменим случайньй потенциал в (4.3) его сужением на $L_{\mathbf{q}} \oplus E_{\mathbf{q}}^{(0)}$ согласно (4.14). Далее на каждом этаже цепной дроби (4.6) выполним замену

$$
\Sigma_{m}(\epsilon, \mathbf{q}) \Rightarrow T_{m}^{\mathbf{q}} \Sigma_{m}(\epsilon, \mathbf{q}) T_{m}^{\mathbf{q}+}+S_{m}^{\mathbf{q}} \Sigma^{\mathrm{eff}}(\epsilon, \mathbf{q}) S_{m}^{\mathbf{q}+}
$$

где

$$
S_{m}^{\mathbf{q}}=P_{m}^{\mathbf{q}}-T_{m}^{\mathbf{q}}
$$

- проектор на ортогональное дополненение к $L_{\mathbf{q}}^{(m)}$ в пространстве $E_{\mathbf{q}}^{(m)}$. Для дальнейшего определим также проектор

$$
S^{\mathbf{q}}=\sum_{m=1}^{n} S_{m}^{\mathbf{q}}
$$

Подстановка (4.16) означает, что точньй учет рассеяния ведется на всех кластерах $\sigma_{m} \subseteq \sigma_{n}^{\max }(m \leq n)$. Влияние других конфигураций учитывается в приближении эффективной среды. В результате на каждом этаже цепной дроби (4.6) суммирование ведется по конечному множеству кластеров $\left\{\sigma_{m}: \sigma_{m} \subseteq \sigma_{n}^{\max }\right\}(m \leq n)$ и, хотя по $\mathbf{i}, \mathbf{j}$ матрицы, входящие в цепную дробь, остаются бесконечными, их обращение становится возможным в аналитическом виде. В частном случае $\alpha=0$ мы получаем аппроксимацию, предложенную в работе [3]. 
В отсутствие ближнего порядка на $m$-м этаже цепной дроби случайный потенциал $\mathcal{U}_{m}(\mathbf{q})$ имеет вид [4]

$$
\mathcal{U}_{m}(\mathbf{q})=\sum_{\mathbf{i} \in \sigma_{m}}\left|\mathbf{i}, \sigma_{m}, \mathbf{q}\right\rangle \Delta \xi\left\langle\mathbf{i}, \sigma_{m}, \mathbf{q}\right|, \quad \alpha=0
$$

При $\alpha \neq 0$ в ортонормированном базисе матричные элементы вида

$$
\left\langle\psi_{\sigma_{m}(\mathbf{l})}, \mathbf{q}\left|\mathcal{U}_{m}(\mathbf{q})\right| \psi_{\sigma_{m}^{\prime}\left(\mathbf{l}^{\prime}\right)}, \mathbf{q}\right\rangle
$$

убывают по степенному закону, если увеличивается расстояние между узлом $\mathbf{0}$ и центрами тяжести кластеров $\mathbf{l}$ и $\mathbf{l}^{\prime}$. Когда параметр $\alpha$ не слишком мал, более целесообразно вместо подстановки (4.14) проводить сужение потенциала с учетом $\alpha$. А именно, на $m$-м этаже цепной дроби определим ортогональные проекторы $R_{m}^{\mathbf{q}}$ на конечное подпространство векторов $\left\{\left|\psi_{\sigma_{m}(\mathbf{l})}, \mathbf{q}\right\rangle\right\}$ таких, что матричные элементы $\left\langle\psi_{\sigma_{m}(\mathbf{l})}, \mathbf{q}\left|\mathcal{U}_{m}(\mathbf{q})\right| \psi_{\sigma_{m}^{\prime}\left(\mathbf{l}^{\prime}\right)}, \mathbf{q}\right\rangle$ имеют порядок по $\alpha$ не старше чем $|\alpha|^{p}$, где $p>0$ - целое число. При этой аппроксимации будем производить замену

$$
\begin{aligned}
\mathcal{U}(\mathbf{q}) \Rightarrow \sum_{m=1}^{n} R_{m}^{\mathbf{q}} \mathcal{U}_{m}(\mathbf{q}) R_{m}^{\mathbf{q}+} & +\sum_{m=1}^{n}\left(T_{m}^{\mathbf{q}} \Delta_{m}(\mathbf{q}) T_{m+1}^{\mathbf{q}+}+T_{m+1}^{\mathbf{q}} \Delta_{m}^{+}(\mathbf{q}) T_{m}^{\mathbf{q}+}\right)+ \\
& +\Delta_{0}(\mathbf{q})+\Delta_{0}^{+}(\mathbf{q}) .
\end{aligned}
$$

\section{5. ПРИБЛИЖЕНИЕ ТИПА БЛУЖДАЮЩЕГО КЛАСТЕРА}

В качестве примера предложенной схемы самосогласования (4.14), (4.16) для массового оператора рассмотрим случай $n=2$, выбрав в качестве максимального кластера $\sigma_{2}^{\max }=\sigma_{2}$ пару ближайших соседей. На втором этаже цепной дроби векторы вида $\left|i, \sigma_{2}, q\right\rangle$ ортогональны друг другу. На первом этаже в ортогональном базисе (4.8) матричные элементы $\widetilde{A}_{i j}(q)=\left\langle\psi_{i}, q\left|A_{q}\right| \psi_{j}, q\right\rangle$ трансляционно-инвариантного оператора $A_{q}$ вида $(4.15)$, которому соответствует матрица $A_{i-j}$, могут быть выражены через свертки

$$
\begin{aligned}
\left\langle i, q\left|A_{q}\right| j, q\right\rangle & =\sum_{k}\langle i, q \mid k, q\rangle A_{k-j}= \\
& =\frac{1}{2 \pi} \int_{-\pi}^{\pi} d p g(p-q) A(p) \exp \{i p(j-i)\}
\end{aligned}
$$

где интегрирование ведется по первой зоне Бриллюэна (постоянная решетки $a=1$ ), $A(p)$ есть фурье-образ матрицы $A_{i-j}$, а $g(q)$ определяется выражением (3.6). Выполнив подстановку (4.14), (4.16) в (4.6) и учитывая $(4.9),(4.10)$, получим

$$
\begin{aligned}
\Sigma_{0}(\epsilon, q) & =\Delta^{2}\left\langle\psi_{0}, q\left|G_{1}^{\mathrm{eff}}(\epsilon, q) \frac{1}{1-W_{1}(\epsilon, q) G_{1}^{\mathrm{eff}}(\epsilon, q)}\right| \psi_{0}, q\right\rangle, \\
W_{1}(\epsilon, q) & =\sum_{i j}\left|\psi_{i}, q\right\rangle W_{i j}^{\sigma_{1}}(\epsilon, q)\left\langle\psi_{j}, q\right|, \quad G_{1}^{\mathrm{eff}}(\epsilon, q)=\left(\epsilon-\mathcal{H}_{1}^{\mathrm{eff}}(\epsilon, q)\right)^{-1} .
\end{aligned}
$$


Отличные от нуля матричные элементы $W_{i j}^{\sigma_{1}}(\epsilon, q)$ имеют вид

$$
\begin{aligned}
& W_{i j}^{\sigma_{1}}(\epsilon, q)=\delta_{i j} \omega_{i}+\Sigma_{i j}^{\sigma_{1}}(\epsilon, q)-\widetilde{\Sigma}_{i j}(\epsilon, q), \quad i, j \in \sigma, \\
& W_{i j}^{\sigma_{1}}(\epsilon, q)=-\widetilde{\Sigma}_{i j}(\epsilon, q), \quad i \in \sigma, \quad j \notin \sigma,
\end{aligned}
$$

здесь

$$
\sigma=\{-1,0,1\}, \quad \omega_{0}=\Delta \xi, \quad \omega_{1}=\omega_{-1}=\frac{\Delta \xi}{1+\alpha} \alpha ;
$$

при $i \notin \sigma, j \in \sigma$ матричный элемент $W_{i j}^{\sigma_{1}}(\epsilon, q)$ получается из соответствующего элемента в (5.3) перестановкой индексов $i$ и $j$. Матричные элементы $\Sigma_{i j}^{\sigma_{1}}(\epsilon, q)$, входящие в первое выражение из (5.3), отличны от нуля при $i, j= \pm 1$ и с учетом (4.12) определяются соотношением, аналогичным (5.2), т.е.

$$
\begin{aligned}
\Sigma_{i j}^{\sigma_{1}}(\epsilon, q)= & b^{2} \exp \{i q(j-i) / 2\} \times \\
& \times\left\langle i / 2, \sigma_{2}, q\left|G_{2}^{\mathrm{eff}}(\epsilon, q) \frac{1}{1-W_{2}(\epsilon, q) G_{2}^{\mathrm{eff}}(\epsilon, q)}\right| j / 2, \sigma_{2}, q\right\rangle, \\
b^{2}= & \frac{b_{1}^{2}}{\Delta^{2}\left(1-\alpha^{2}\right)}, \\
W_{2}(\epsilon, q)= & \sum_{i j}\left|i, \sigma_{2}, q\right\rangle W_{i j}^{\sigma_{2}}(\epsilon, q)\left\langle j, \sigma_{2}, q\right|, \\
G_{2}^{\mathrm{eff}}(\epsilon, q)= & \left(\epsilon-\mathcal{H}_{2}^{\mathrm{eff}}(\epsilon, q)\right)^{-1} .
\end{aligned}
$$

Здесь $\sigma_{2}=\{-1 / 2,1 / 2\}, W_{i j}^{\sigma_{2}}(\epsilon, q)=0$, если $i, j \notin \sigma_{2}$, в другом случае

$$
W_{i j}^{\sigma_{2}}(\epsilon, q)=v_{0} \delta_{i j}-\Sigma_{i-j}(\epsilon), \quad v_{0}=\frac{\Delta \xi}{1+\alpha} .
$$

Следуя работе [3], все матрищы, входящие в (5.4), представим в блочной форме

$$
\left[\begin{array}{ll}
A_{1} & A_{3} \\
A_{3}^{\mathrm{T}} & A_{2}
\end{array}\right],
$$

где $i, j \in \sigma_{2}$ для $\left(A_{1}\right)_{i j} ; i \in \sigma_{2}, j \notin \sigma_{2}$ для $\left(A_{3}\right)_{i j} ; i, j \notin \sigma_{2}$ для $\left(A_{2}\right)_{i j}$; символ ${ }^{\mathrm{T}}$ обозначает операцию транспонирования. При таком разбиении блок $W_{2}$ равен 0 . Используя формулы обрашения блочных матриц [9], получим

$$
\begin{aligned}
& \Sigma^{\sigma_{1}}(\epsilon, q)=b^{2} \exp \{i q(j-i) / 2\} \frac{1}{\Gamma^{-1}(\epsilon, q)-\left(v_{0}-\Sigma_{1}+\Sigma_{3} G_{2}^{\text {eff }} \Sigma_{3}^{\mathrm{T}}\right)}, \\
& \Gamma^{-1}(\epsilon, q)=\left(1+\Sigma_{3} G_{3}^{\mathrm{eff}^{\mathrm{T}}}\right)\left(G_{1}^{\mathrm{eff}}\right)^{-1}\left(1+G_{3}^{\mathrm{eff}} \Sigma_{3}^{\mathrm{T}}\right),
\end{aligned}
$$

где индексы $1,2,3$ используются для обозначения блоков $(5.5)$ матриц $\Sigma_{i-j}(\epsilon)$ и $G_{i-j}(\epsilon)$. Для вычисления (5.6) удобно определить операторы [10]

$$
\begin{aligned}
& G^{\mathrm{eff}}(\epsilon, q)=(\epsilon-\mathcal{H}(\epsilon, q))^{-1}, \quad I^{(1)}(\epsilon, q)=G^{\mathrm{eff}}(\epsilon, q) \Sigma^{\mathrm{eff}}(\epsilon, q), \\
& I^{(2)}(\epsilon, q)=\Sigma^{\mathrm{eff}}(\epsilon, q) G^{\mathrm{eff}}(\epsilon, q) \Sigma^{\mathrm{eff}}(\epsilon, q)
\end{aligned}
$$


Тогда в выражения (5.6) входят матрицы размера $(2 \times 2)$, определяемые матричными элементами операторов (5.7) вида $G_{i-j}^{\mathrm{eff}}(\epsilon), \Sigma_{i-j}(\epsilon), I_{i-j}^{(1)}(\epsilon), I_{i-j}^{(2)}(\epsilon)(i, j= \pm 1 / 2)$.

Аналогично можно выполнить обрашение матрицы, входящей в (5.2). Получающееся в результате выражение для $\Sigma_{0}(\epsilon, q)$ имеет вид, формально совпадаюший с (5.6). Отличие состоит в том, что матришы вида (5.5) определены в ортогональном базисе (4.7) и разбиение на блоки определяется относительно кластера $\sigma$. Соответственно в выражении, аналогичном $(5.6)$, матрицы размера $(3 \times 3)$ составлены из величин $\widetilde{\Sigma}_{i j}(\epsilon, q)$, $\widetilde{G}_{i j}(\epsilon, q), \widetilde{I}_{i j}^{(1)}(\epsilon, q), \widetilde{I}_{i j}^{(2)}(\epsilon, q)(i, j=0, \pm 1)$, представляюших собой матричные элементы операторов (5.7) в ортогональном базисе. В результате получаем самосогласованное нелинейное интегральное уравнение, определяющее $\Sigma_{0}(\epsilon, q)$, которое необходимо решать численно. В отсутствие ближнего порядка $(\alpha=0)$ предложенная аппроксимация совпадает с приближением блуждаюшего кластера $[3,11]$.

\section{6. ОБОБЩЕНИЕ ДЛЯ СЛУЧАЯ ПРОИЗВОЛЬНЫХ КОРРЕЛЯЦИЙ И РАЗМЕРНОСТИ}

Вычисление многоузельных коррелящионных функций в реальных системах является сложной задачей. В дальнейшем будем полагать, что нам заданы необходимые корреляционные функции. Пусть базисный вектор | vac $\rangle$ (3.2) по-прежнему порождает одномерное пространство $E_{0}$. Определим векторы $\left|\sigma_{m}(\mathbf{l})\right\rangle, m>0$, ортогональные к $\mid$ vac $\rangle$, следующим образом:

$$
c_{\sigma_{m}}\left|\sigma_{m}(\mathbf{l})\right\rangle=\left(\prod_{\mathbf{i} \in \sigma_{m}(\mathbf{l})} \hat{\xi}_{\mathbf{i}}\right)|\mathrm{vac}\rangle+a_{\sigma_{m}}|\mathrm{vac}\rangle
$$

где коэффициент $a_{\sigma_{m}}$ определяется из условия $\left\langle\operatorname{vac} \mid \sigma_{m}(\mathbf{l})\right\rangle=0$, а константа $c_{\sigma_{m}}$ - из условия нормировки вектора $\left|\sigma_{m}(\mathbf{l})\right\rangle$. Добавляя электронные функции аналогично (3.16), получим набор векторов $\left|\mathbf{i}+\mathbf{1}, \sigma_{n}(\mathbf{l})\right\rangle$, которые образуют полный базис в $\Psi \otimes \Phi$. Гамильтониан $\mathcal{H}$, действуюший на расширенном пространстве $\Psi \otimes \Phi$, в узельном представлении по-прежнему имеет вид (3.15). Используя (4.2) и переходя к импульсному представлению, запишем гамильтониан $\mathcal{H}(\mathbf{q})$ в виде $(4.3)$. Пусть $E_{\mathbf{q}}^{(0)}$ - одномерное пространство, порожденное вектором $\left|\mathbf{0}, \sigma_{0}, \mathbf{q}\right\rangle$. Обозначим через $E_{\mathbf{q}}$ линейную оболочку векторов $\left\{\left|\mathbf{i}, \sigma_{m}, \mathbf{q}\right\rangle\right\}$ таких, что $\sigma_{m} \subseteq \sigma_{n}^{\max }(m>0)$, т.е. $E_{\mathbf{q}}$ является пространством кластеров, рассеяние на которых будем учитывать точно. Пусть $P_{0}^{\mathbf{q}}$ - ортогональный проектор на пространство $E_{\mathbf{q}}^{(0)}, P_{\mathbf{q}}^{(1)}$ - ортогональный проектор на $E_{\mathbf{q}}, Q_{0}^{\mathbf{q}}=1-P_{0}^{\mathbf{q}}$, $Q_{\mathbf{q}}^{(1)}=1-P_{0}^{\mathbf{q}}-P_{\mathbf{q}}^{(1)}$. Определим также операторы

$$
\begin{gathered}
\Delta_{0}(\mathbf{q})=P_{0}^{\mathbf{q}} \mathcal{U}(\mathbf{q}) Q_{0}^{\mathbf{q}}, \quad \Delta^{(1)}(\mathbf{q})=P_{\mathbf{q}}^{(1)} \mathcal{U}(\mathbf{q}) Q_{\mathbf{q}}^{(1)} \\
\mathcal{H}^{(1)}(\mathbf{q})=P_{\mathbf{q}}^{(1)} \mathcal{H}(\mathbf{q}) P_{\mathbf{q}}^{(1)}
\end{gathered}
$$

Усредненная функция Грина $G_{0}(\epsilon, \mathbf{q})$ гамильтониана $(1.1)$ определяется выражением (4.4). Поскольку теперь пространства, соответствующие кластерам с различным 
числом узлов, не ортогональны друг другу, то представление массового оператора в виде цепной дроби неудобно. Поэтому запишем точное выражение для $\Sigma_{0}(\epsilon, \mathbf{q})$ в виде

$$
\Sigma_{0}(\epsilon, \mathbf{q})=\Delta_{0}(\mathbf{q}) \frac{1}{\epsilon-\mathcal{H}^{(1)}(\mathbf{q})-\Sigma^{(1)}(\epsilon, \mathbf{q})} \Delta_{0}^{+}(\mathbf{q})
$$

где

$$
\Sigma^{(1)}(\epsilon, \mathbf{q})=\Delta^{(1)}(\mathbf{q}) \frac{1}{\epsilon-Q_{\mathbf{q}}^{(1)} \mathcal{H}(\mathbf{q}) Q_{\mathbf{q}}^{(1)}} \Delta^{(1)+}(\mathbf{q}) .
$$

Пусть $T^{\mathbf{q}}$ есть ортогональный проектор на пространство $L_{\mathbf{q}}$ векторов $\left|\mathbf{i}, \sigma_{m}, \mathbf{q}\right\rangle$ таких, что $\mathbf{i} \cup \sigma_{m} \subseteq \sigma_{n}^{\max }(m>0)$. Согласно (6.1) и (4.3) оператор $\mathcal{U}(\mathbf{q})$ отображает пространство $L_{\mathbf{q}}$ на $L_{\mathbf{q}} \oplus E_{\mathbf{q}}^{(0)}$. Поскольку $\mathcal{U}(\mathbf{q})$ эрмитов, то из (6.3) следует, что

$$
T^{\mathbf{q}} \Sigma^{(1)}(\epsilon, \mathbf{q})=\Sigma^{(1)}(\epsilon, \mathbf{q}) T^{\mathbf{q}}=0 \text {. }
$$

Возможные аппроксимации для $\Sigma_{0}(\epsilon, \mathbf{q})$ состоят в следующем. Сделаем в гамильтониане (4.3) замену (4.14) и положим в $(6.2) \Sigma^{(1)}(\epsilon, \mathbf{q})=0$. Получим несамосогласованное выражение для $\Sigma_{0}(\epsilon, \mathbf{q})$. Для получения самосогласованного уравнения, кроме сужения случайного потенциала на $L_{\mathbf{q}} \oplus E_{\mathbf{q}}^{(0)}$, выполним в (6.2) подстановку, удовлетворяющую соотношению (6.4):

$$
\Sigma^{(1)}(\epsilon, \mathbf{q}) \Rightarrow S^{\mathbf{q}} \Sigma^{\mathrm{eff}}(\epsilon, \mathbf{q}) S^{\mathbf{q}}
$$

где

$$
S^{\mathbf{q}}=P_{\mathbf{q}}^{(1)}-T^{\mathbf{q}}
$$

a $\Sigma^{\mathrm{eff}}(\epsilon, \mathbf{q})$ определяется соотношением (4.15). Предложенная схема самосогласования совпадает для марковской цепочки с ранее рассмотренной схемой и при отсутствии корреляций между случайными величинами переходит в аппроксимацию, предложенную в [3].

Как и прежде, представим матрицы, входящие в (6.2), в блочной форме (5.5), где разбиение на блоки производится относительно пространства $L_{\mathbf{q}}$. Определение $\Sigma_{0}(\epsilon, \mathbf{q})$ приводит к выражению, аналогичному (5.6). Его вычисление сводится к перемножению и обрашению матриц конечных размеров, которые, подобно (5.1), составлены из величин

$$
\begin{aligned}
& \left\langle\mathbf{i}, \sigma_{n}, \mathbf{q}\left|A_{\mathbf{q}}\right| \mathbf{j}, \sigma_{m}^{\prime}, \mathbf{q}\right\rangle= \\
& \quad=\frac{1}{(2 \pi)^{d}} \int d \mathbf{p} g^{\sigma_{n} \sigma_{m}^{\prime}}(\mathbf{p}-\mathbf{q}) A(\mathbf{p}) \exp \{i \mathbf{p}(\mathbf{j}-\mathbf{i})\}
\end{aligned}
$$

где $A_{\mathbf{q}}$ - оператор вида $(4.15), A(\mathbf{p})$ - фурье-образ матрицы $A_{\mathbf{i}-\mathbf{j}}$, а $g^{\sigma_{n} \sigma_{m}^{\prime}}(\mathbf{p}-\mathbf{q})-$ фурье-образ матрицы Грама скалярного произведения векторов $\left|\sigma_{m}(\mathbf{i})\right\rangle$, определенных B $(6.1)$ :

$$
g_{\mathbf{i} j}^{\sigma_{m}} \sigma_{k}^{\prime}=\left\langle\sigma_{m}(\mathbf{i}) \mid \sigma_{k}^{\prime}(\mathbf{j})\right\rangle
$$


Интегрирование в (6.6) выполняется по первой зоне Бриллюэна рассматриваемой решетки, $d$ - размерность пространства. В итоге получаем интегральное уравнение для $\Sigma_{0}(\epsilon, \mathbf{q})$.

\section{7. ПРЕДЕЛЬНЫЕ СЛУЧАИ УПОРЯДОЧЕННОГО КРИСТАЛЛА}

Одним из преимушеств представления усредненной резольвенты, рассмотренного в этой работе, является то, что если положить параметр Каули равным критическому, то в предложенных приближениях мы получим правильные предельные выражения для функции Грина. Для примера рассмотрим двумерную квадратную решетку и смоделируем корреляционную функцию между парой атомов, находящихся в узлах решетки $\mathbf{r}_{1}$ и $\mathbf{r}_{2}$, следующим образом:

$$
g_{\mathbf{r}_{1} \mathbf{r}_{2}}=\left\langle\hat{\xi}_{\mathbf{r}_{1}} \hat{\xi}_{\mathbf{r}_{2}}\right\rangle=\Delta^{2} \alpha^{\left\|\mathbf{r}_{1}-\mathbf{r}_{2}\right\|},
$$

где $\left\|\mathbf{r}_{1}-\mathbf{r}_{2}\right\|=\left|x_{1}-x_{2}\right|+\left|y_{1}-y_{2}\right|, \quad|\alpha|<1-$ параметр Каули. Тогда при $\alpha=1$ сплав распадается на две подсистемы, состояшие из атомов только сорта $\mathbf{A}$ или только сорта В. При конщентрации $x=0.5$ и $\alpha=-1$ атомы $\mathbf{A}$ и $\mathbf{B}$ чередуются на решетке. В этих случаях точные выражения для функций Грина известны и мы можем их сравнить с выражениями нашей работы.

Для фурье-образа матрицы $g_{\mathbf{r}_{1} \mathbf{r}_{2}}(7.1)$ при $\alpha= \pm 1$ имеем формулы

$$
\begin{array}{ll}
g(\mathbf{q})=(2 \pi)^{2} \sum_{\mathbf{g}} \delta(\mathbf{q}+\mathbf{g}), & \alpha=1, \\
g(\mathbf{q})=(2 \pi)^{2} \sum_{\mathbf{g}} \delta(\mathbf{q}+\mathbf{Q}+\mathbf{g}), & \alpha=-1,
\end{array}
$$

здесь суммирование выполняется по всем векторам $\mathbf{g}$ обратной решетки, $\mathbf{Q}=(\pi, \pi)$ (постоянная решетки $a=1)$. Обозначим через $|\mathbf{i}, \mathbf{q}\rangle=|(n k), \mathbf{q}\rangle$ базисные векторы пространства одноузельных возбуждений в фурье-представлении. Ортогонализуем их, используя (7.1):

$$
\begin{aligned}
&\left|\phi_{(00)}, \mathbf{q}\right\rangle=|\mathbf{0}, \mathbf{q}\rangle \\
&\left|\phi_{(n k)}, \mathbf{q}\right\rangle=\left(1-\alpha^{2}\right)^{-1}\left(|(n k), \mathbf{q}\rangle-\alpha \exp \left\{i q_{2}\right\}|(n, k-1), \mathbf{q}\rangle-\right. \\
&\left.-\alpha \exp \left\{i q_{1}\right\}|(n-1, k), \mathbf{q}\rangle+\alpha^{2} \exp \left\{i q_{1}+i q_{2}\right\}|(n-1, k-1), \mathbf{q}\rangle\right), \\
& n>0, \quad k>0, \quad\left(q_{1}, q_{2}\right)=\mathbf{q} .
\end{aligned}
$$

Для других случаев $n$ и $k$ имеем аналогичные формулы. При $n=0$ или при $k=0$ базис ортогонализуется согласно (4.7).

Сопоставим матрише интегралов перескока $V_{\mathbf{i}-\mathbf{j}}$ оператор $V_{\mathbf{q}}$, действуюший в расширенном пространстве так же, как и в формулах (4.15). Поскольку векторы, соответствующие многократным возбуждениям, можно сделать ортогональными векторам $\left|\phi_{(n k)}, \mathbf{q}\right\rangle$, то при $\alpha= \pm 1$ достаточно рассмотреть матричные элементы $V_{\mathbf{q}}$ вида 
$\left\langle\mathbf{0}, \mathbf{q}\left|V_{\mathbf{q}}\right| \phi_{(n k)}, \mathbf{q}\right\rangle$. Используя (6.6), (7.2), (7.3), получаем

$$
\begin{aligned}
& \widetilde{V}_{00}(\mathbf{q})=\left\langle\mathbf{0}, \mathbf{q}\left|V_{\mathbf{q}}\right| \mathbf{0}, \mathbf{q}\right\rangle=V(\mathbf{q}), \quad \alpha=1, \\
& \widetilde{V}_{00}(\mathbf{q})=\left\langle\mathbf{0}, \mathbf{q}\left|V_{\mathbf{q}}\right| \mathbf{0}, \mathbf{q}\right\rangle=V(\mathbf{q}+\mathbf{Q}), \quad \alpha=-1, \\
&\left\langle\mathbf{0}, \mathbf{q}\left|V_{\mathbf{q}}\right| \phi_{(n k)}, \mathbf{q}\right\rangle \rightarrow \frac{1-\alpha}{1+\alpha} V(\mathbf{q}) \exp \left\{i q_{1} n+i q_{2} k\right\} \rightarrow 0, \quad \alpha \rightarrow 1, \\
&\left\langle\mathbf{0}, \mathbf{q}\left|V_{\mathbf{q}}\right| \phi_{(n k)}, \mathbf{q}\right\rangle \rightarrow \frac{1+\alpha}{1-\alpha} V(\mathbf{q}+\mathbf{Q}) \exp \left\{i\left(q_{1}+\pi\right) n+i\left(q_{2}+\pi\right) k\right\} \rightarrow 0, \\
& \alpha \rightarrow-1 .
\end{aligned}
$$

Следовательно, в предельных случаях матрица $\mathcal{H}^{(1)}(\mathbf{q})$ в $(6.2)$ факторизуется, и для усредненной функции Грина имеем выражение

$$
\begin{aligned}
& G_{0}(\epsilon, \mathbf{q})=\frac{1}{\epsilon-\bar{\epsilon}-V(\mathbf{q})-\Sigma_{0}(\epsilon, \mathbf{q})}, \\
& \Sigma_{0}(\epsilon, \mathbf{q})=\frac{\Delta^{2}}{\epsilon-\bar{\epsilon}-\widetilde{V}_{00}(\mathbf{q})-\Delta \xi},
\end{aligned}
$$

где константы $\Delta, \Delta \xi$ и $\bar{\epsilon}$ определены в (2.6) и (2.4).

В случае $\alpha=1$ выражение для усредненной резольвенты имеет вид

$$
G_{0}(\epsilon, \mathbf{q})=\frac{x}{\epsilon-\epsilon_{\mathbf{A}}-V(\mathbf{q})}+\frac{y}{\epsilon-\epsilon_{\mathbf{B}}-V(\mathbf{q})},
$$

что совпадает с точным значением функции Грина сплава, распавшегося на две подсистемы с весами $x$ и $y$.

В случае $\alpha=-1$ и $x=0.5$, используя (7.4), для диагонального матричного элемента усредненной резольвенты получим выражение

$$
\bar{G}_{00}(\epsilon)=\frac{1}{(2 \pi)^{2}} \int d \mathbf{q} \frac{\epsilon-\bar{\epsilon}-V(\mathbf{q}+\mathbf{Q})}{\left(\epsilon-\epsilon_{\mathbf{A}}\right)\left(\epsilon-\epsilon_{\mathbf{B}}\right)-(\epsilon-\bar{\epsilon})(V(\mathbf{q})+V(\mathbf{q}+\mathbf{Q}))+V(\mathbf{q}) V(\mathbf{q}+\mathbf{Q})},
$$

которое совпадает с точным выражением для функции Грина идеального кристалла с расположением атомов $\mathbf{A}$ и $\mathbf{B}$ на квадратной решетке в шахматном порядке.

\section{8. АНАЛИТИЧЕСКИЕ СВОЙСТВА РЕЗОЛЬВЕНТЫ И ЕЕ АППРОКСИМАЦИЙ}

Резольвента самосопряженного оператора $\mathcal{H}$ обладает свойствами [12]

$$
G^{+}(\epsilon)=G\left(\epsilon^{*}\right), \quad G\left(\epsilon_{1}\right)-G\left(\epsilon_{2}\right)=\left(\epsilon_{2}-\epsilon_{1}\right) G\left(\epsilon_{1}\right) G\left(\epsilon_{2}\right),
$$

важным следствием которых является знакоопределенность ее антиэрмитовой части (свойство Герглотца). $G_{0}(\epsilon)$ в отличие от $G(\epsilon)$ не удовлетворяет второму уравнению из (8.1), однако свойство Герглотца для нее выполняется и вытекает из условия

$$
P_{0} G(\epsilon) G^{+}(\epsilon) P_{0} \geq 0 .
$$


Любая аппроксимация резольвенты $G_{0}(\epsilon)$, для которой

$$
\begin{aligned}
G^{+}(\epsilon) & =G\left(\epsilon^{*}\right), \\
G_{0}\left(\epsilon_{1}\right)-G_{0}\left(\epsilon_{2}\right) & =\left(\epsilon_{2}-\epsilon_{1}\right) G_{0}\left(\epsilon_{1}\right) \Phi\left(\epsilon_{1}, \epsilon_{2}\right) G_{0}\left(\epsilon_{2}\right),
\end{aligned}
$$

где $\Phi\left(\epsilon_{1}, \epsilon_{2}\right)$ - операторная аналитическая функция двух переменных, удовлетворяющая условию $\Phi\left(\epsilon, \epsilon^{*}\right) \geq 0$, обладает знакоопределенной антиэрмитовой частью, т.е.

$$
G_{0}(\epsilon)-G_{0}^{+}(\epsilon)=\left(\epsilon^{*}-\epsilon\right) G_{0}(\epsilon) \Phi\left(\epsilon, \epsilon^{*}\right) G_{0}^{+}(\epsilon) .
$$

Несамосогласованные аппроксимации для $\Sigma_{0}(\epsilon, \mathbf{q})$, получаемые обрывом цепной дроби (4.6) на $m$-м этаже, удовлетворяют условию (8.2) и, следовательно, обладают правильными аналитическими свойствами. Покажем теперь, что процедура самосогласования типа (4.14), (4.16) для одномерной цепочки и схема $(4.14),(6.5)$ для общего случая дают уравнение на $\Sigma_{0}(\epsilon, \mathbf{q})$, удовлетворяюшее условию (8.2). Обозначим

$$
G^{(1)}(\epsilon, \mathbf{q})=\left[\epsilon-P_{\mathbf{q}}^{(1)} \mathcal{H}_{\mathrm{vc}}(\mathbf{q}) P_{\mathbf{q}}^{(1)+}-T^{\mathbf{q}} \mathcal{U}(\mathbf{q}) T^{\mathbf{q}+}-S^{\mathbf{q}} \Sigma^{\mathrm{eff}}(\epsilon, \mathbf{q}) S^{\mathbf{q}+}\right]^{-1},
$$

где проекторы $T^{\mathbf{q}}, S^{\mathbf{q}}$ и $P_{\mathbf{q}}^{(1)}$ определены в разделах 4 и 6 , оператор $\Sigma^{\mathrm{eff}}(\epsilon, \mathbf{q})$ имеет вид (4.15). Тогда, используя (4.6) или (6.2), находим

$$
\begin{aligned}
& G_{0}(\epsilon, \mathbf{q})-G_{0}\left(\epsilon^{*}, \mathbf{q}\right)=\left(\epsilon^{*}-\epsilon\right) G_{0}(\epsilon, \mathbf{q})\left(1+\frac{\Delta \Sigma_{0}(\mathbf{q})}{\epsilon^{*}-\epsilon}\right) G_{0}^{+}(\epsilon, \mathbf{q}), \\
& \Delta \Sigma_{0}(\mathbf{q})=\Delta_{0}(\mathbf{q}) G^{(1)}(\epsilon, \mathbf{q})\left(\epsilon^{*}-\epsilon+S^{\mathbf{q}} \Delta \Sigma^{\mathrm{eff}}(\mathbf{q}) S^{\mathbf{q}+}\right) G^{(1)+}(\epsilon, \mathbf{q}) \Delta_{0}^{+}(\mathbf{q}),
\end{aligned}
$$

где

$$
\Delta \Sigma_{0}(\mathbf{q})=\Sigma_{0}(\epsilon, \mathbf{q})-\Sigma_{0}\left(\epsilon^{*}, \mathbf{q}\right), \quad \Delta \Sigma^{\mathrm{eff}}(\mathbf{q})=\Sigma^{\mathrm{eff}}(\epsilon, \mathbf{q})-\Sigma^{\mathrm{eff}}\left(\epsilon^{*}, \mathbf{q}\right) .
$$

Рассмотрим далее некоторое абстрактное линейное пространство $\Theta$, в котором выбран полный ортонормированный базис $\{|\phi(\mathbf{1}, \sigma)\rangle\}$, где $\sigma \subseteq \sigma_{n}^{\max }, \sigma \neq \sigma_{0}$, а индекс $\mathbf{l}$ пробегает все узлы решетки. Обозначим через $|\phi(\mathbf{q}, \sigma)\rangle$ фурье-образы векторов $|\phi(\mathbf{l}, \sigma)\rangle$, определенные соотношением, аналогичным (4.2). Тогда имеем

$$
S^{\mathbf{q}} \Delta \Sigma^{\mathrm{eff}}(\mathbf{q}) S^{\mathbf{q}+}=\sum_{\mathbf{p}} S^{\mathbf{q}} K^{\mathbf{q}} \Delta \Sigma_{0}(\mathbf{p}) \hat{g}^{-1}(\mathbf{p}, \mathbf{q}) K^{\mathbf{q}+} S^{\mathbf{q}+},
$$

где

$$
K^{\mathbf{q}}=\sum_{\mathbf{l}, \sigma \subseteq \sigma_{n}^{\max }}|\mathbf{l}, \sigma, \mathbf{q}\rangle\langle\phi(\mathbf{l}, \sigma)|,
$$

а оператор $\hat{g}^{-1}(\mathbf{p}, \mathbf{q})$ есть обратный к оператору

$$
\hat{g}(\mathbf{p}, \mathbf{q})=\sum_{\sigma, \sigma^{\prime} \subseteq \sigma_{n}^{\max }}|\phi(\mathbf{p}, \sigma)\rangle g^{\sigma \sigma^{\prime}}(\mathbf{p}-\mathbf{q})\left\langle\phi\left(\mathbf{p}, \sigma^{\prime}\right)\right|,
$$


здесь $g^{\sigma \sigma^{\prime}}(\mathbf{p}-\mathbf{q})$ - фурье-образ матрицы Грама (6.7). Поскольку матрица Грама положительно определена, то оператор $\hat{g}^{-1}(\mathbf{p}, \mathbf{q})$ также положительно определен. Структура уравнений (8.3), (8.4) показывает, что любая итерация уравнения (8.3) обладает свойством Герглотца, если им обладает начальное условие. Если итерационный процесс $(8.3),(8.4)$ сходится, то его предел дает усредненную резольвенту с требуемыми аналитическими свойствами.

\section{9. ЗАКЛЮЧЕНИЕ}

Нами предложен метод, который в рамках формализма расширенного пространства позволяет учитьвать корреляции в расположении атомов. Используя этот подход, мы получили выражение в виде бесконечной цепной дроби для усредненной резольвенты в случае одномерной цепочки с корреляциями марковского типа. В этом представлении проанализированы различные аппроксимации и показано, что дополнительным к имеюшемуся в теории [4] малому параметру является параметр Каули $\alpha$. Предложены схемы, которые обобшают метод на системы любой размерности и на любые наперед заданные корреляционные функции.

Достоинством развитого в статье подхода является то, что в рассматриваемых приближениях он допускает предельные переходы, когда параметр Каули $\alpha$ стремится к критическому. При этом усредненная функция Грина совпадает с точной для упорядоченного кристалла. При $\alpha=0$ мы получаем выражения известных ранее аппроксимаций $[3,4]$.

Анализ аналитических свойств приближений показьвает, что они удовлетворяют свойству Герглотца, тем самым обеспечивая положительную плотность состояний во всем интервале энергий.

Работа выполнена при частичной финансовой поддержке Российского фонда фундаментальных исследований, грант № 97-02-16270.

\section{Список литературы}

[1] A. Mookerjee. J. Phys. C. 1973. V. 6. № 8. P. 1340-1349.

[2] A. Mookerjee. J. Phys. C. 1973. V. 6. № 10. P. L205-L208.

[3] T. Kaplan, P. L. Leath, L.J. Gray, H.W. Diehl. Phys. Rev. B. 1980. V. 21. № 10. P. $4230-4246$.

[4] А.К. Аржников, С. Г. Новокионов. ТМФ. 1990. Т. 84. № 1. С. 128-140.

[5] L. J. Gray, T. Kaplan. Phys. Rev. B. 1981. V. 24. № 4. P. 1872-1882.

[6] А.К. Аржииков, Л. В. Добьиева, С. Г. Новокшонов. ФММ. 1993. Т. 76. № 5. С. 32-39.

[7] T. Saha, I. Dasgupta, A. Mookerjee. J. Phys.: Cond. Matt. 1994. V. 6. P. L245-L251.

[8] В. Феллер. Введение в теорию вероятностей и ее приложения. Т. І. М.: Мир, 1967.

[9] Ф. Р. Гантмахер. Теория матриц. М.: Наука, 1988.

[10] A.K. Arzhnikov, L. V. Dobisheva, S. G. Novokshonov. J. Phys: Cond. Matt. 1991. № 3. P. $9025-9032$.

[11] R. Mills, P. Ratanavararaksa. Phys. Rev. B. 1978. V. 18. № 10. P. 5291-5308.

[12] М. Рид, Б. Саймон. Методы современной математической физики. Т. 1. М.: Мир, 1977. 